\title{
Vasovagal Syncope: A Review of Current and Future Strategies
}

\author{
Melanie Dani, ${ }^{1,2}$ Dimitrios Panagopoulos, ${ }^{1}$ Andreas Dirksen, ${ }^{1}$ Patricia Taraborrelli, ${ }^{1}$ Miriam Torocastro, ${ }^{1}$ Richard Sutton ${ }^{1,3}$ \\ and Phang Boon Lim ${ }^{1}$ \\ 1. Imperial Syncope Unit, Imperial College Healthcare NHS Trust, Hammersmith Hospital, London, UK; 2. Cutrale Perioperative and Ageing Group, Department
} of Bioengineering, Imperial College London, London, UK; 3. National Heart and Lung Institute, Imperial College London, London, UK

DOI: https://doi.org/10.17925/EJAE.2021.7.1.40

V asovagal syncope is common, and while it is usually associated with a benign prognosis, it results in significant physical and psychological morbidity for affected individuals. Despite comprehensive understanding of the underlying pathophysiology, there have been few definitive therapeutic advances until recent randomized, controlled trials in pharmacotherapy, pacing and cardioneuroablation. In this review, we highlight the increasing recognition that the condition is multifactorial, and emphasize and discuss multifaceted management approaches, including education, psychological wellbeing, dietary and fluid intake, pharmacotherapy and cardiac intervention.

\section{Keywords}

Vasovagal syncope, reflex, pacing, neuroablation, midodrine

Disclosures: Melanie Dani, Dimitrios Panagopoulos, Andreas Dirksen, Patricia Taraborrelli, Miriam Torocastro, Richard Sutton and Phang Boon Lim have no financial or non-financial relationships or activities to declare in relation to this article.

Review process: Double-blind peer review

Compliance with ethics: All procedures were

followed in accordance with the responsible committee on human experimentation and with the Helsinki Declaration of 1975 and subsequent revisions, and informed consent was received from the patient involved in the case study included in this review.

Authorship: All named authors meet the criteria of the International Committee of Medical Journal Editors (ICMJE) for authorship for this manuscript, take responsibility for the integrity of the work as a whole and have given final approval for the version to be published.

Access: This article is freely accessible at touchCARDIO.com (c) Touch Medical Media 2021

Received: 24 August 2020

Accepted: 13 January 2021

Published online: 28 July 2021

Citation: European Journal of Arrhythmia

\& Electrophysiology. 2021;7(1):40-7

Corresponding author: Melanie Dani, Imperial Syncope Unit, Hammersmith Hospital, Imperia College Healthcare NHS Trust, Ducane Road,

London, W12 OHS, UK. E: melanie.dani@nhs.net

Support: No funding was received in

the publication of this article.
Syncope - a rapid onset, transient loss of consciousness with spontaneous complete recovery, and resulting from cerebral hypoperfusion - is common. ${ }^{1}$ With a cumulative lifetime incidence of $35-50 \%$, syncope accounts for $1-3 \%$ of emergency department visits. ${ }^{2-4}$ vasovagal syncope (VVS) is the most common cause of syncope. ${ }^{4}$ While WV is usually associated with a benign prognosis, it is linked with notable morbidity and psychological distress, particularly if there is a delay in diagnosis. ${ }^{5}$ Also, its prognosis may not be as benign as has been assumed, as it appears to carry an increased likelihood of cardiovascular disease in later life. ${ }^{6}$ Recurrent VVS is associated with impaired quality of life, and functional impairment similar to other chronic diseases such as back pain and rheumatoid arthritis. ${ }^{7.8}$ Psychosocial impairment, somatisation, anxiety and depression are common. ${ }^{8}$

Despite advances in the understanding of the pathophysiology of VVS, significant uncertainty remains. Individuals with WV represent a heterogenous patient population, and different mechanisms are likely to contribute. These mechanisms are still not fully understood. Therefore, determining dominant mechanisms and modifying therapies accordingly is not always possible. In this review, we first describe the pathophysiology of the condition and discuss an approach for evaluating patients with WV, emphasising holistic, multifaceted care. We also discuss current and emerging therapies. While some practical, conservative measures remain effective and are recommended, newer interventions, such as pacing and ganglionic plexus ablation, may bring relief for some patients.

\section{Pathophysiology of vasovagal syncope}

When a healthy person stands up, gravitational forces cause 500-1000 mL of blood to pool in the pelvic, abdominal and leg vessels, resulting in reduced venous return to the heart. Baroreceptor firing in the carotid sinus increases dynamically in response to reduced stretch of the vessel wall caused by a fall in arterial pressure. This results in increased sympathetic tone and withdrawal of parasympathetic (vagal) tone, which is mediated by the nucleus solitarius in the brainstem. As a result, the heart rate increases, and peripheral vasoconstriction occurs. Blood pressure (BP) recovers, or is maintained, allowing the individual to continue standing.9-13

VVS is a neural reflex involving the autonomic nervous system. This is manifested by the vagally mediated Bezold-Jarisch reflex. In response to reduced filling of the left ventricle or the right atrium by reduced venous return (due to venous pooling in the splanchnic bed), parasympathetic activity is increased and sympathetic activation is decreased. A triad of bradycardia, hypotension and altered respiratory pattern ensues. ${ }^{12}$ Four stages have been described below and are illustrated in Figure $1 .{ }^{10}$

1. An early stabilization phase after standing, when lower body venous pooling occurs, resulting in decreased ventricular filling followed by compensatory vasoconstriction to increase systemic vascular resistance. Cardiac output is maintained or slightly falls. 
1.

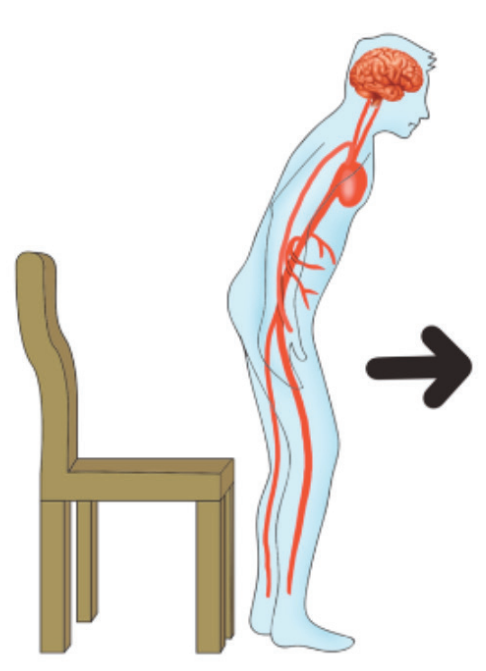

3.

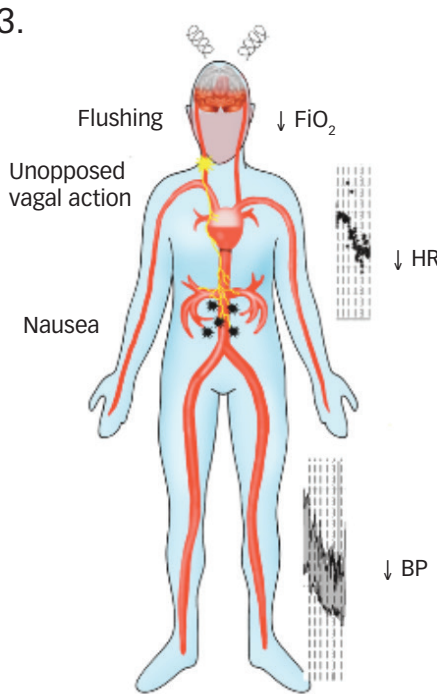

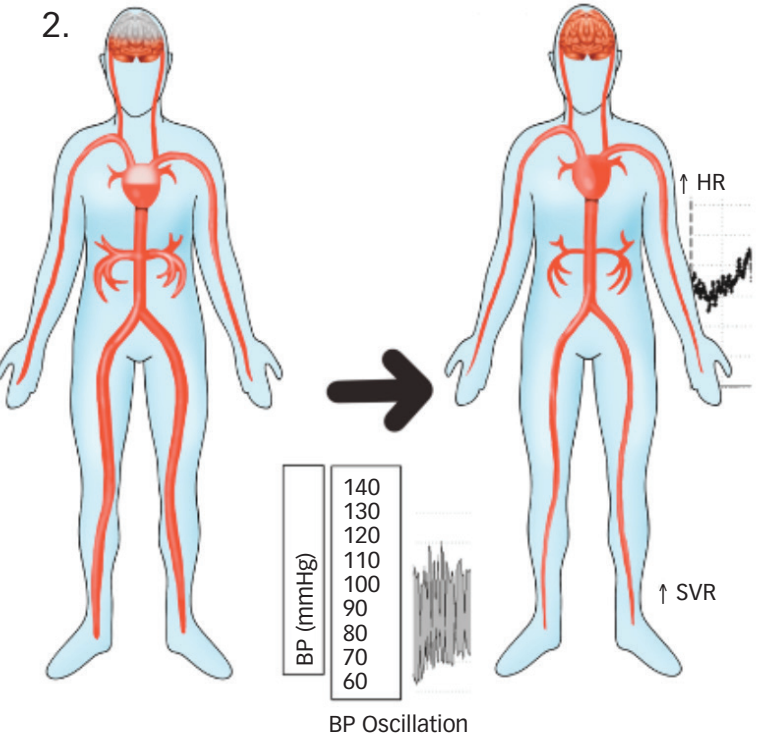

4.
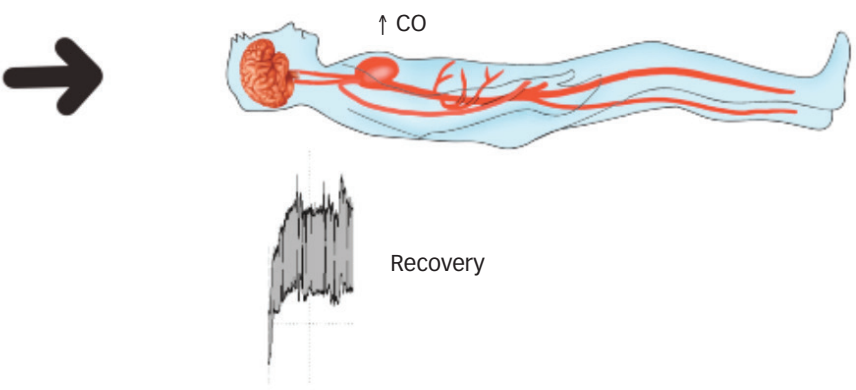

$\uparrow \mathrm{BP}$

The four stages of VVS: 1. Venous pooling in the lower body on standing, resulting in decreased ventricular filling and compensatory vasoconstriction. 2. BP begins to fall but compensatory vascular resistance rises; oscillating BP may be seen. 3. Cardioinhibition and greater hypotension ensues, resulting in syncope. 4. Recovery, characterized by increased venous return to the heart, increased cardiac output and restoration of cerebral perfusion.

$\mathrm{BP}=$ blood pressure; $\mathrm{CO}=$ cardiac output; $\mathrm{FiO}_{2}=$ fraction of inspired oxygen; $\mathrm{HR}=$ heart rate; $\mathrm{SVR}=$ systemic vascular resistance; $\mathrm{VVS}=$ vasovagal syncope.

2. Circulatory instability occurs when cardiac output falls slightly but compensatory systemic vascular resistance rises; in this period there may be oscillation of $\mathrm{BP}$, suggesting a failing or stressed servo-mechanism. This period is dominated by falling BP known as vasodepression.

3. Terminal hypotension and syncope, which is marked by a sudden fall in BP due to the onset of cardioinhibition and usually preceded by sympathetic withdrawal, possibly related to the well-recognized epinephrine peak beginning to fall. With cardioinhibition (vagal influence), the heart rate falls steeply even to asystole. The duration of vasodepression is approximately 9 minutes before onset of cardioinhibition. ${ }^{14}$ During this phase, autonomic symptoms such as flushing and nausea may be expected. Pancreatic polypeptide levels rise in the terminal phase, probably as a marker of vagal activity and relates to nausea or vomiting. This change may continue into recovery. ${ }^{15}$ The point at which syncope occurs depends on the speed and extent of hypotension and cerebral autoregulation.
It is known that cerebral oxygenation begins to fall early in stage 2 and is $<60 \%$ at syncope when the BP is usually $<60 \mathrm{mmHg}$. ${ }^{16}$

4. The final stage is recovery; when the individual is supine after losing consciousness, venous return increases by the gravitational change draining the splanchnic pooling into the right heart, rapidly improving preload, stroke volume and cardiac output to provide resumption of cerebral perfusion (Figure 1). ${ }^{10-12}$

The different heart rate and BP behaviours during orthostasis can be classified into collapse patterns, which are described and later modified by the Vasovagal Syncope International Study (VASIS). ${ }^{17}$ The intention was to be able to correctly target any therapy. Unfortunately, this has proved less reliable than hoped. Nevertheless, the VASIS classification remains widely used in practice. The most common form (type 1) is a mixed pattern of vasodepression and cardioinhibition. Type 2 describes patterns dominated by cardioinhibition. Type $2 \mathrm{~A}$ is less severe than $2 \mathrm{~B}$, which includes 
asystole. Type 3 is a pattern in which vasodepression dominates and heart rate fall is present but minimal.

Neuroendocrine activity plays a part in the reflex probably as a protective mechanism and begins with vasopressin release, followed by epinephrine. High epinephrine levels have a vasodilatory effect on skeletal muscle, favouring some fall in BP.10,12 Other neurotransmitters that can modulate neuroendocrine status include serotonin, adenosine and opioids. ${ }^{12}$

\section{Evaluation}

When assessing an individual with suspected WS, a comprehensive history and witness account are crucial for making a diagnosis. A detailed description of the circumstances around the event should be elicited, including the ambient environment, temperature, provoking factors, posture at the time of syncope (and the duration of that posture) and the individual's general health and hydration status at the time. Autonomic symptoms prior to syncope, such as sensations of warmth, nausea, light-headedness, visual dimming and auditory dimming, are very common. Previous episodes, along with medical history and medication history should be recorded. Care should be taken to consider a differential diagnosis, asking specifically about lateral tongue bite, prolonged loss of consciousness and prolonged confusion after the episode, which suggest a seizure. Full details are reported together with the potential for a definite diagnosis by this approach in $>90 \%$ of those presenting. ${ }^{3.18}$ Absent or very brief prodrome, or injury suggesting lack of warning, should prompt exclusion of arrhythmia or structural heart disease. However, brief or absent prodromes occur in older subjects with WV mostly due to retrograde amnesia. ${ }^{19}$ Detailed physical examination and electrocardiogram should be performed to exclude - as far as possible - structural heart disease, although echocardiography may be needed in some.

Tilt table tests are valuable for confirming the diagnosis. ${ }^{1}$ In common with most medical tests, sensitivity and specificity are not perfect, so a negative tilt table test does not exclude WVS. ${ }^{20,21}$ However, a recent meta-analysis showed a discriminatory ability to detect VVS, with an odds ratio of 12.5 compared with asymptomatic individuals.22 Recent European Society of Cardiology (ESC) guidelines recommend the use of tilt table testing in WV (Class 2A) for:

- confirming a diagnosis of reflex syncope (as well as other orthostatic intolerance syndromes) when suspected, but not confirmed;

- educating and empowering the patient to recognize symptoms and appreciate the effects of counter pressure manoeuvres; and

- to recognize a 'hypotensive susceptibility' in individuals with unexplained syncope, albeit acknowledging that this may not explain the full mechanism of syncope and thus not make a full diagnosis. Recognition of 'hypotensive susceptibility' is of great value when pacing is being considered as, if it exists, future syncope recurrences will be more frequent. ${ }^{1,23}$

Implantable loop recorders (ILRS) are indicated when structural heart disease is excluded and another diagnosis is uncertain from the initial evaluation, with or without tilt testing. In our practice, tilt testing is often chosen before ILR because of our familiarity with the test and its favourable economic outcome. This does not exactly follow the advice of the ESC guidelines. ${ }^{1}$ A modified strategy based on those guidelines is that described by Brignole et al. in the Syncope Unit 2 project. ${ }^{23}$

\section{Belief, hope and expectation of recovery: Tools for empowerment and improved compliance}

A strong benefit of tilt table testing is the ability to clearly demonstrate the BP and heart rate changes in the patient on a beat-to-beat basis, correlating these changes with the patient's own experience of clinical symptoms. In our experience of over 6,000 tilt testing procedures (unpublished), one of the recurring themes from our patients relates to beliefs. In our tertiary syncope centre, we are often referred patients who have undergone multiple investigations and who have seen colleagues in neurology, cardiology and general internal medicine. These patients have developed the inappropriate belief that some of their pre-syncopal symptoms, such as chest pain, palpitations and shortness of breath, may be due to a sinister, potentially life-threatening cause, which is yet to be discovered. This may be because the investigations until this point have been normal. They believe that an important diagnosis has been missed, which may directly contribute to harm and distress and have a major impact on the patient's wellbeing. ${ }^{5}$ Their beliefs may be enhanced by family members who have witnessed attacks and been very frightened by what they have seen, as the subject may have appeared dead or had abnormal jerky movements that could be misinterpreted as epilepsy.

In these instances, patients who attend for tilting and who receive a full explanation of their clinical experience, anchored by their tilt data, are immediately able to accept and believe that all their symptoms, including the perceived 'sinister' ones, can be explained by BP and heart rate fluctuations. Once the patient accepts and comprehends the diagnosis, our clinical tilt team enforces expectation of complete recovery, through an understanding of the mechanisms underscoring the symptoms, and (usually) conservative management strategies. Thus, hope is instilled; perhaps the most important but overlooked component of successful patient recovery, because with hope comes a shift in outlook pointing towards recovery, manifested by improved compliance and, ultimately, better health.

Figure 2 shows the tilt tracing of a 28-year-old female patient whose presentation was atypical of VVS. Within the first 6 minutes of being head-up tilted, she started to experience palpitations and chest pain, and within 8 minutes she experienced shortness of breath. These symptoms worsened throughout the passive phase of tilting, and eventually VVS occurred, with a VASIS 1 pattern (mixed vasodepressor and cardioinhibitory response). Symptoms included light-headedness and dizziness, with loss of vision 30 seconds before syncope. Our patient's symptom profile of daily episodes of palpitations, shortness of breath and chest pain, with rarer monthly episodes of near-syncope/ syncope, were recognized by her as being reproduced on tilt; for her this resulted in full reassurance. The findings were demonstrable for the patient to see on the recordings, with sympathetic activation resulting in sinus tachycardia, tachypnoea and probable atypical chest pain from increased cardiac inotropy and chronotropy. Thus, this tilt table test was highly beneficial and reassuring to the patient in revealing the physiological changes to explain the symptoms. Furthermore, she could see the helpful effects of using counter-pressure manoeuvres at symptom onset to reverse the changes. There are strikingly few data on the positive psychological benefits of tilt testing, and this may be an area of future research in identifying effective therapies.

\section{Management}

\section{Overall principles of management}

As a result of the psychosocial impairment and distress caused by recurrent syncope, and the resultant loss of quality of life, an empathetic, holistic, patient-based approach is a core tenet of care.,5 There is a paucity of definitive high-quality evidence for management, so individualized multidisciplinary care is essential. A summary of recommendations is shown in Table 1. 
Figure 2: Tilt trace showing a young woman with vasovagal syncope

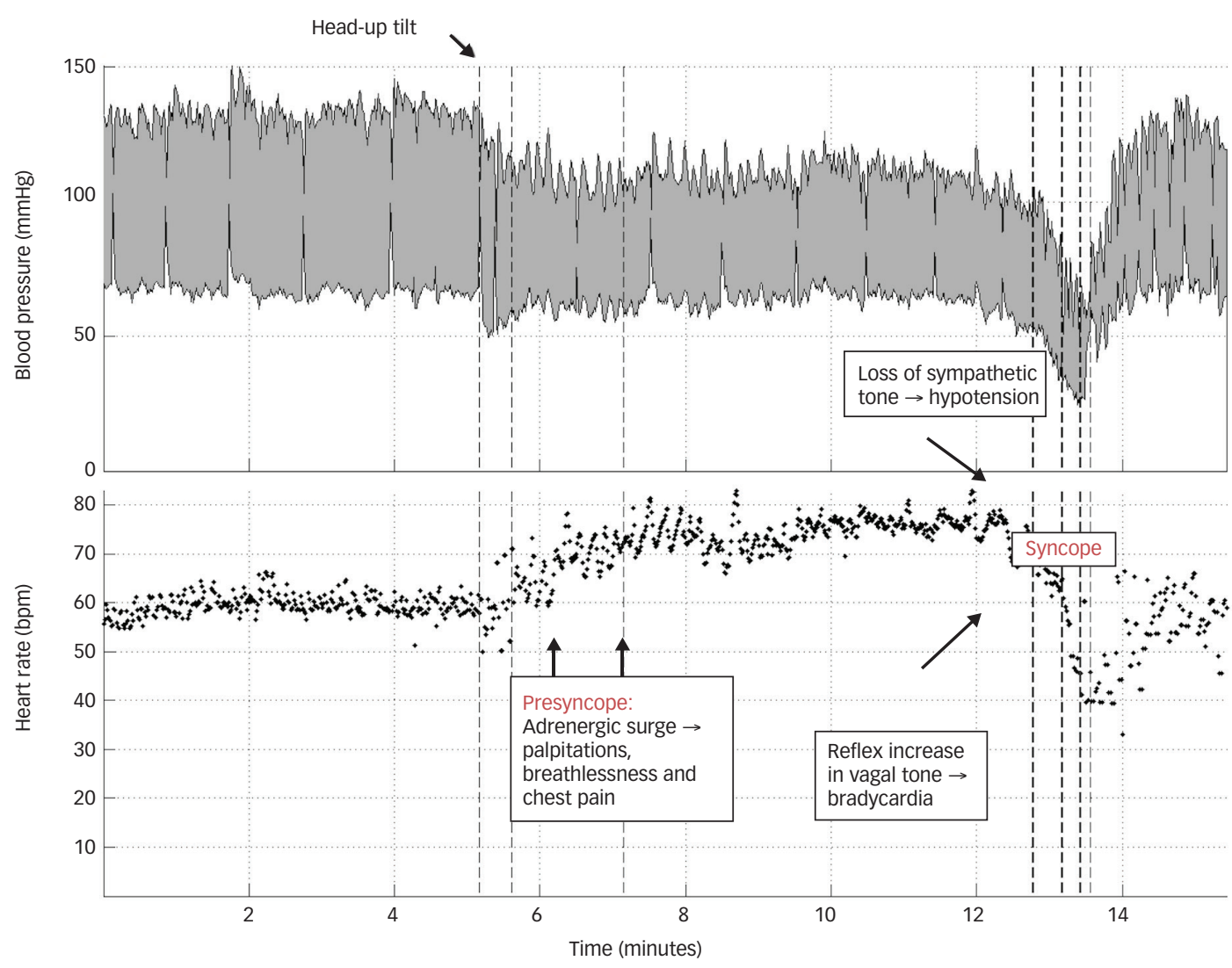

A 28-year-old woman who presented with recurrent episodes of palpitations, chest pain and syncopal episodes underwent a head-up tilt test. After 6 minutes, she developed her typical symptoms of palpitations, anxiety and chest pain. These correlate with the pre-syncopal phase and adrenergic surge, with a compensatory tachycardia. These symptoms worsened, with shortness of breath at 8 minutes until syncope occurred at 13 minutes, which resulted from an abrupt withdrawal of sympathetic tone (resulting in hypotension) and a reflex augmentation in parasympathetic tone (resulting in bradycardia).

Table 1: Practical management measures and educational aspects in vasovagal syncope

\begin{tabular}{|c|c|}
\hline Management aspect & Examples \\
\hline \multicolumn{2}{|l|}{ Education } \\
\hline Explain mechanism of symptoms and how they relate to the patient & Ideally using animations or illustration of mechanisms \\
\hline $\begin{array}{l}\text { Educate on evasive counter-pressure manoeuvres (tilt table testing } \\
\text { may help illustrate this) }\end{array}$ & $\begin{array}{l}\text { Crossing legs, clenching lower body muscles (e.g. thighs, buttocks), squatting, leaning forward } \\
\text { and folding arms }\end{array}$ \\
\hline $\begin{array}{l}\text { Establish any situations where safety may be compromized and } \\
\text { encourage avoidance }\end{array}$ & Going to a sauna alone, standing upright in enclosed spaces \\
\hline $\begin{array}{l}\text { Identify provoking factors and postures and advise avoidance } \\
\text { of these }\end{array}$ & $\begin{array}{l}\text { Warmth, dehydration, alcohol. } \\
\text { If having medical procedures, warn nurses and ensure couch or bed available }\end{array}$ \\
\hline Reassurance & $\begin{array}{l}\text { Reassure that with compliance to above measures, complete recovery is expected and } \\
\text { prognosis is excellent }\end{array}$ \\
\hline Driving advice & $\begin{array}{l}\text { Category } 1 \text { private vehicles: if WVS occurs while standing, or when sitting and is } \\
\text { precipitated by an avoidable trigger (which will not be present on driving), they may drive and } \\
\text { need not notify the DVLA. If there is not an avoidable trigger, they must not drive until the } \\
\text { annual risk of recurrence is }<20 \% \text {. } \\
\text { Category } 2 \text { commercial vehicles: the patient must not drive and must contact the DVLA }\end{array}$ \\
\hline Lifestyle measures & Ensure hydration, and aim for water and salt repletion \\
\hline \multicolumn{2}{|l|}{ Interventions } \\
\hline Medications & $\begin{array}{l}\text { Medications may have a role if lifestyle measures are ineffective and symptoms are very } \\
\text { severe }\end{array}$ \\
\hline Pacemaker implantation and cardioneuroablation & $\begin{array}{l}\text { Consider in selected patients with minimal prodrome, increasing risk of injury, and with } \\
\text { documented cardioinhibition }\end{array}$ \\
\hline
\end{tabular}

DVLA = Driver and Vehicle Licensing Agency; VVS = vasovagal syncope. 


\section{Education}

Comprehensive multidisciplinary rehabilitation programmes for recurrent WS have shown significant improvements in health-related quality of life, fewer recurrent syncopal episodes, plus fewer medical consultations, hospitalizations and traumatic injuries, compared with standard care. ${ }^{24,25}$ Such programmes include reassurance and education about the benign prognosis of the condition, education about physical counter-manoeuvres, and exercise training. Some programmes include dietary advice about hydration and salt intake, and psychological care.

Even enrolling in a clinical trial improves health-related quality of life in those with recurrent VVS, independent of recurrent syncopal events. ${ }^{26}$ This may be due to enhanced wellbeing from interaction with healthcare providers, a reflection of the individual's motivation to self-manage their condition or a psychological adjustment to the diagnosis.

Patient advocacy groups providing patient and staff education (e.g. the Syncope Trust and Reflex Anoxic Seizures [STARS], an enterprise of the Arrhythmia Alliance) provide freely available information to help empower patients. ${ }^{.}$The authors of this article provide freely accessible patient education resources at www.stopfainting.com.

Staff education is also important, particularly in clinical areas where WV may be precipitated, such as phlebotomy or minor surgery clinics. Simple instructional videos can train staff to be competent in recognizing and managing the condition. ${ }^{27}$

Finally, once the diagnosis of VVS is established, and if the individual drives a vehicle, an assessment on road safety should be made. In the UK, if the syncope occurred on standing, category 2 commercial vehicle drivers (bus, lorry, etc.) must not drive and must notify the Driver Vehicle and Licensing Authority (DVLA). If the VVS occurred on standing, then the indivudual must not drive for 3 months, and investigations into the cause should be considered. For category 1 private vehicle drivers (car and motorbike), the individual can continue to drive and does not need to contact the DVLA, provided the VVS occurs only on standing. If the syncope occurred on sitting, the prodrome was of sufficient duration to allow the driver to stop safely before loss of consciousness and was triggered by something that will not be present when driving, then they can continue to drive. If there is no clear trigger or the prodrome was unreliable, they must contact the DVLA; additionally, guidelines advise that the individual must not drive until their clinician's estimated annual risk of recurrence is less than $20 \%{ }^{28}$

\section{Key messages:}

1. Pre-syncopal symptoms include not only lightheadedness and dizziness, but also breathlessness, chest pain and palpitations.

2. Tilt table testing can be an invaluable way of demonstrating that both presyncopal and syncopal symptoms are due to heart rate and BP fluctuations.

\section{Medication review}

Many older patients with VVS are hypertensive and require hypotensive drugs; however, it has to be borne in mind that most hypotensive agents exaggerate vasodepression and may therefore increase attacks. There is evidence that patients' symptoms can be reduced or even abolished by reducing or ceasing the use of these agents. ${ }^{29}$ ESC guidelines recommend an individualized approach to antihypertensive medications, but suggest reducing or stopping therapy in patients with hypotensive susceptibility - essentially vasodepression - aiming for a systolic target of $140 \mathrm{mmHg} .{ }^{1}$ There are other frequently prescribed medications that have hypotensive effects, which also should be reviewed.

\section{Physical counter-manoeuvres}

At the onset of pre-syncopal symptoms, lower body tensing (clenching of leg, buttock and abdominal muscles together with concomitant leg crossing, plus linking of the fingers and pulling apart vigorously without letting go) results in an almost instantaneous increase in mean arterial pressure, stroke volume and cardiac output, raising the BP and reducing syncope frequency. ${ }^{30}$ These measures are less effective in older persons, where buttock-clenching is the sole measure that may help..$^{31}$ These manoeuvres work when they are performed at the onset of symptoms, so they are of no benefit to individuals who lack warning symptoms. The ESC guidelines have, on the basis of these trials, recommended them if the individual is under 60 years old and has a sufficiently long prodrome.?

\section{Water and salt supplementation}

A single $500 \mathrm{~mL}$ bolus of water can increase $\mathrm{BP}$, total peripheral resistance and orthostatic tolerance. ${ }^{32}$ The effect is thought to be from a vasopressor response. This technique has been shown to be helpful in individuals at risk of VVS; for example, those undergoing blood donation. ${ }^{33}$ Salt and water repletion are suggested as 'reasonable' in European and American guidelines. ${ }^{1,34}$ Caution should be exercised in those with heart failure, renal failure and urinary continence issues.

\section{Pharmacological trials}

\section{Beta-adrenergic receptor blockers}

Beta-adrenergic receptor blockers (beta blockers) are postulated to work via inhibiting left ventricular mechanoreceptors, resulting in decreased adrenergic tone and countering adrenaline-mediated vasodilatation. However, double-blinded, randomized, controlled trials have not shown benefits of atenolol or propranolol in preventing WVS, compared to placebo. ${ }^{35,36}$ Metoprolol was studied in the Prevention of Syncope Trial (POST-1) study, but failed in its primary endpoint of preventing syncope recurrence. ${ }^{37}$ However, when post-hoc analyses stratified participants by age, there was a significant benefit in individuals over 42 years old. ${ }^{38} \mathrm{~A}$ randomized, controlled trial, the POST-5 study, is currently underway to assess efficacy in individuals over 40 years. ${ }^{39}$

The ESC guidelines suggest that there is sufficient evidence to advise against beta blockers for prevention of recurrent WS, while American guidelines state that they may be useful in individuals over 42 years old. ${ }^{1,34}$

\section{Volume expanders: Fludrocortisone}

Fludrocortisone is a volume expander which increases sodium and water absorption. Although it failed to reach its primary endpoint in the second Prevention of Syncope trial (POST-2), there was a significant benefit in individuals who were stabilised with a dose of 200 mcg per day. ${ }^{40}$ ESC guidelines suggest that it can be of benefit in younger people with normal or low blood pressure and no comborbidities, and the American college of Cardiology guidelines advise that it may be useful in individuals who have not responded to salt and fluid repletion. ${ }^{1,34}$ Fludrocortisone is often poorly tolerated, with side effects including weight gain.

\section{Alpha-adrenoceptor agonists}

Midodrine is a peripherally acting alpha-2 adrenoceptor agonist, which increases BP by venous and arterial vasoconstriction. ${ }^{41} \mathrm{~A}$ randomized, controlled trial has shown that it is beneficial in individuals with tilt-positive frequent syncope, compared with standard care. ${ }^{42} \mathrm{~A}$ 
small uncontrolled study suggested that it is effective when other pharmacological treatments are unsuccessful. ${ }^{43}$ However, in individuals who remained symptomatic despite non-pharmacological management, there was no benefit from midodrine. ${ }^{44}$ ESC guidelines suggest that in view of contrasting data and small studies not reaching criteria for a clinical trial, alpha-agonists may be effective, but they urge further research. 'American guidelines make similar recommendations, but highlight the important contraindications of hypertension, heart failure and urinary retention, mostly in males. ${ }^{34}$

\section{Norepinephrine reuptake transporter inhibitors}

Norepinephrine reuptake transporter (NET) inhibitors increase the levels of noradrenaline in sympathetic synapses, thus increasing sympathetic tone, reducing the potential for vagal reflex, and antagonizing sympathetic withdrawal. In the Prevention of Syncope Trial 6 - Atomoxetine in Vasovagal Syncope (POST 6), atomoxetine significantly reduced the risk of tilt-induced syncope. The proposed mechanism was by attenuating reflex bradycardia. ${ }^{45}$

A meta-analysis of four studies examining NET inhibitors (reboxetine, sibutramine and atomoxetine) found that taking NET inhibitors resulted in a lower risk of WV in healthy volunteers, and that atomoxetine reduced recurrent episodes in patients with VVS. ${ }^{46}$ These studies post-date guidelines; therefore, they can be prescribed only on an individual basis ${ }^{1,34}$

\section{Selective serotonin reuptake inhibitors}

Serotonin may contribute to the sympathetic withdrawal reflex in VVS. Selective serotonin reuptake inhibitors result in the downregulation of serotonin receptors in the brainstem, and could theoretically attenuate this response. ${ }^{47}$ Early and very small studies suggest that sertraline and fluoxetine could be of benefit in individuals with recurrent syncope who are intolerant of other medications. ${ }^{48,49}$ These agents are not recommended in ESC guidance, but American guidance suggests that they 'might be considered'. ${ }^{1,34}$

Key message: Conservative measures are an essential tenet of treatment, and patients should be encouraged to incorporate them into their daily routines. There may be a role for drug therapy in selected patients in whom conservative measures are not sufficient.

\section{Non-interventional studies currently underway}

Researchers realise the multifactorial nature of the condition, and that a multifaceted approach is required. This is reflected by the variety of ongoing clinical trials in syncope prevention registered on www.clinicaltrials.gov, examining the effects of drugs (midodrine, atomoxetine, metoprolol and droxidopa), psychological wellbeing (hypnosis, psychotherapy and yoga) and the management of comorbidity (sleep apnoea). One randomized, controlled study is underway in England, examining whether an iliac arteriovenous anastomosis results in increased venous preload to the heart on standing, abolishing VVS (ClinicalTrials.gov identifier: NCT02388087). ${ }^{50}$

\section{Interventional approaches}

As described earlier, WS results from altered autonomic activation in response to mechanoreceptor and baroreceptor stimulation, mainly by reducing volume within the heart, large vessels and lungs, which is vasodepression. This eventually paradoxically causes parasympathetic activation, which leads to cardioinhibition (bradycardia) and sympathetic withdrawal. The VASIS classification of collapse patterns on tilt table testing was aimed at tailoring individual therapy to different autonomic responses; however, over time, it has become clear that, although useful, it falls short of its target. ${ }^{17}$ The two main interventions that can be offered under certain conditions are pacemaker therapy and cardioneuroablation (CNA).

\section{Pacemaker therapy}

Pacemaker therapy in VVS remains controversial. Dual chamber pacemakers with rate drop response (RDR) or closed loop stimulation (CLS) algorithms have been suggested as potential treatment options

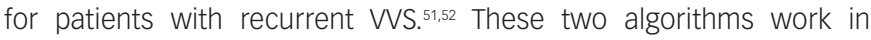
different ways, determined by the sensing or detection system. RDR detects a falling heart rate, which is not precipitous - as in sudden atrioventricular block -, but it is limited because it must wait for the onset of cardioinhibition, which is almost always preceded by vasodepression. ${ }^{14} \mathrm{CLS}$ detects reducing right ventricular volume (and possibly increasing right ventricular contractility) to trigger pacing using the change in impedance to a signal between the electrode in the right ventricular and the can of the pulse generator. Once triggered, the two algorithms behave similarly by increasing the prevailing heart rate by dual chamber stimulation. It is possible that CLS commences therapy sufficiently early in VVS to ameliorate the falling stroke volume. It certainly delivers pacing therapy substantially before RDR. ${ }^{53}$

Use of CLS pacing has been subject to a number of trials, including the spain trial, which showed a benefit for a broader spectrum of patients than those selected by ILR findings in the International Study of Syncope of Unexplained Etiology (ISSUE-3) RDR study. ${ }^{21,54}$ The BIOSync study has recently reported on CLS pacing, adding qualification of its importance. ${ }^{55}$ These matters have been fully discussed in a review in the Heart Rhythm journal, although another review of the subject provides a slightly different perspective. ${ }^{56,57}$ The story of trials in pacing VS stretches over 20 years and remains controversial, as implanted devices over a lifetime from youth to old age is an unattractive choice especially when clear evidence of benefit from a well-designed trial was lacking until 2012. ${ }^{54}$ Despite the potential benefit being evident, there rightly remains conservatism, as expressed by sud et al. ${ }^{58}$

An unexpected finding of the ISSUE-3 study was that patients who were tilt-positive benefitted little from pacing, while those who were tilt-negative with the same ILR findings received benefit in terms of a great reduction in syncope recurrence after pacing. ${ }^{54}$ This revelation prompted an attempt to explain the phenomenon on the basis of tilt induction of hypotensive susceptibility, which may be relatively hidden by cardioinhibition, and was followed by the ESC guidelines on pacing and inspired the Syncope Unit Project (SUP)-2.23,59,60 For older patients likely presenting with reflex syncope, a protocol was employed that started with carotid sinus massage. If positive with cardioinhibition, patients received dual chamber RDR pacing. If negative, patients proceeded to have a tilt test; if this revealed cardioinhibition, they were then treated as above. If negative or positive with vasodepression, an ILR was inserted. If the ILR findings later showed cardioinhibition, these patients were also paced by similar devices. Follow-up showed that syncope recurrence was rare in the carotid-sinus-positive group, but was relatively common in patients who were tilt-positive and those ultimately paced on the basis of ILR findings. These results were interpreted as confirmation of the hypotensive susceptibility raised by Sutton and Brignole, although the data were more encouraging for the use of pacing in reducing the frequency of syncope recurrence than the trial data from ISSUE-3; this implies that patients of this type require counselling concerning a reduction in syncope, rather than its 
abolition. ${ }^{23,54,59}$ Whether CLS pacing would have yielded better results is currently unknown.

There are no available trial data for pacemaker therapy in individuals younger than 40 years of age. In these patients, first-line management should always be a conservative approach with measures mentioned previously. Therefore, pacemaker therapy should only be considered in a highly selected group of older patients where lack of prodrome is a prominent feature, such as those in the ISSUE-3 study, providing a means to prevent traumatic injury and, at least, a reduction in frequency of attacks.

\section{Cardioneuroablation}

Cardiac autonomic denervation was initially undertaken by endocardial identification of epicardial ganglionated plexi and their ablation, building upon neuromodulation targets for atrial fibrillation with encouraging early results. ${ }^{61,62}$ Subsequently, there has been limited but growing evidence that cardioneuroablation may help patients with severely symptomatic recurrent VVS with a pronounced asystolic component. ${ }^{63,64}$

The heart's intrinsic autonomic innervation consists of a complex network of ganglia and nerves that lie on the epicardial surface usually embedded within the epicardial fat. Clusters of these nerves forming the ganglionated plexi have been shown to play a significant role in the autonomic neuromodulation of the heart innervating the atrioventricular node and all four pulmonary veins.65,66 These clusters are essentially parasympathetic, post-ganglionic neurons that are amenable to catheter ablation due to their location. These have been located not only anatomically through histological studies, but also functionally by means of high-frequency stimulation $(20 \mathrm{~Hz})$ during endocardial catheter ablation procedures. ${ }^{66,67}$ High-frequency stimulation of the ganglionated plexi produces a significant vagal response, leading to reduced heart rate (defined by an $R-R$ interval increase of $>50 \%$ from baseline or transient atrioventricular block).

Pachon et al. recruited 43 patients with dominant cardioinhibitory response on head-up tilt and refractory episodes of WV despite optimal medical treatment. ${ }^{68}$ Using spectral mapping and anatomical position of ganglionated plexi, they proceeded with catheter radiofrequency ablation as they had in their original description. Of the 43 patients, 40 did not experience further syncope for the duration of follow-up (range 11-91 months). ${ }^{68}$
Yao et al. recruited 10 patients with a median of $3.5 \mathrm{VVS}$ episodes in the preceding year (range 2-20) and a positive head-up tilt test. ${ }^{64}$ Ganglionated plexi were identified using high-frequency stimulation and targeted intracardially using catheter radiofrequency ablation. None of the patients had recurrence of syncope for the duration of follow-up (range 13-55 months). ${ }^{64}$ Sun et al., from the same Beijing group, recruited 57 patients with refractory VVS (failed conservative treatment measures and two positive head-up tilts with at least one episode of syncope in the preceding 6 months). ${ }^{69}$ They proceeded with ganglionated plexi ablation, following the same protocol as Yao et al. ${ }^{64}$ Of the patients recruited, $91.2 \%$ were free from syncope at 102 months' follow up. It is important to note that these studies have all been pre-post studies (also known as before-and-after studies), with no controls or randomization.

In summary, there are encouraging results from systematic case series' data in support of CNA. A highly selected, very symptomatic group of patients with dominant cardioinhibitory VVS could benefit from CNA, by either completely abolishing syncope, or extending the prodrome long enough to prevent injury. Due to a paucity of data, ESC guidelines, at present, have no specific recommendation concerning CNA. More data are needed in the form of randomized, controlled trials, together with an agreement on which ganglionated plexi should be ablated.

Key message: It may be appropriate to consider pacemaker therapy and cardioneuroablation in a selected group of patients with confirmed dominant cardioinhibition and insufficient prodrome to prevent injury.

\section{Conclusion}

VVS is a very common condition, which, although it may be benign, results in significant psychological and psychosocial morbidity. Management should take a multidisciplinary, multifaceted approach with education and lifestyle management playing a critical role. Educating patients about their symptoms by reproducing those symptoms on tilt table testing can be invaluable reassurance. VVS is a topic that is currently inspiring active, ongoing research, with promising findings emerging from pacing and CNA in selected patients. Clinicians, researchers and patients should be fully informed of all management strategies to allow truly holistic and individualized care. $\square$
1 Brignole M, Moya A, de Lange FJ, et al. 2018 ESC guidelines for the diagnosis and management of syncope. Eur Heart $J$. for the diagnosis and

2. Ganzeboom KS, Mairuhu G, Reitsma JB, et al. Lifetime cumulative incidence of syncope in the general population: study of 549 Dutch subjects aged 35-60 years. J Cardiovasc Electrophysiol. 2006;17:1172-6.

3. Wieling W, Thijs RD, Linzer M, et al. Great expectations: what patients with unexplained syncope desire. J Intern Med. 2016;279:259-64.

4. Kenny RA, Bhangu J, King-Kallimanis BL. Epidemiology of syncope/collapse in younger and older Western patient populations. Prog Cardiovasc Dis. 2013;55:357-63.

5. Lobban TCA. Syncope: a patient and family perspective. Cardiol Clin. 2013:31:1-8.

6. Ricci F, Sutton R, Palermi S, et al. Prognostic significance of non-cardiac syncope in the general population: a systematic non-cardiac syncope in the general population: a systemat.
review and meta-analysis. I Cardiovasc Electrophysiol. review and meta-an

7. Rose MS, Koshman ML, Spreng S, Sheldon R. The relationship between health-related quality of life and frequency of spells in patients with syncope. J Clin Epidemiol. 2000;63:1209-16.

8. Linzer M, Pontinen M, Gold DT, et al. Impairment of physical and psychosocial function in recurrent syncope. J Clin Epidemio 1991;44:1037-43.

9. Freeman R, Abuzinadah AR, Gibbons $C$, et al. Orthostatic hypotension: JACC state-of-the-art review. J Am Coll Cardiol. 2018;72:1294-309.

10. Jardine $\mathrm{DL}$, Wieling $\mathrm{W}$, Brignole $\mathrm{M}$, et al. The pathophysiology of the vasovagal response. Heart Rhythm 2018.15:921-9.

11. Wieling W, Jardine DL, de Lange FJ, et al. Cardiac output and vasodilation in the vasovagal response: an analysis of the classic papers. Heart Rhythm. 2016;13:798-805.

12. Fenton AM, Hammill SC, Rea RF, et al. Vasovagal syncope. Ann Intern Med. 2000;133:714-25.

13. Kaufmann $\mathrm{H}$, Norcliffe-Kaufmann $\mathrm{L}$, Palma JA. Baroreflex dysfunction. N Eng/ J Med. 2020;382:163-78.

14. van Dijk JG, Ghariq M, Kerkhof Fl, et al. Novel methods for quantification of vasodepression and cardioinhibition during tilt-induced vasovagal syncope. Circ Res. 2020;127:e126-38.

15. Fitzpatrick $A$, Williams TR, Ahmed $R$, et al. Echocardiograph and endocrine changes during vasovagal syncope induced by prolonged head-up tilt. Eur I Card Pacing Electrophysiol. 1992:2:121-8.

16. Bachus $\mathrm{E}$, Holm H, Hamrefors $\mathrm{V}$, et al. Monitoring of cereba oximetry during head-up tilt test in adults with history of syncope and orthostatic intolerance. Europace. 2018;20:1535-42.

17. Brignole M, Menozzi C, Del Rosso A, et al. New classification of haemodynamics of vasovagal syncope: beyond the VASIS classification. Europace. 2000;2:66-76.

18. Wieling W, van Dijk N, de Lange FJ, et al. History taking as a diagnostic test in patients with syncope: developing expertise in syncope. Eur Heart J. 2015;36:277-80.

19. O'Dwyer C, Bennett $K$, Langan $Y$, et al. Amnesia for loss of consciousness is common in vasovagal syncope. Europace. 2011;13:1040-5.

20. Petersen MEV, Williams TR, Gordon C, et al. The normal response to prolonged passive head-up tilt testing Heart. 2000;84:509-14.

21. Baron-Esquivias G, Diaz Martin AJ, Del Castillo AM, et al. Head-up tilt test diagnostic yield in syncope diagnosis. J Electrocardiol. 2020;63:46-50

22. Forleo C, Guida P, lacoviello M, et al. Head-up tilt testing for diagnosing vasovagal syncope: a meta-analysis. Int $\mathrm{J}$ Cardiol 2013;168:27-35

23. Brignole M, Arabia F, Ammirati F, et al. Standardised algorithm for cardiac pacing in older patients affected by severe unpredictable reflex syncope: 3-year insights from the syncope Unit Project 2 (SUP2) study. Europace. 2016;18:1427-33.

24. Simeon E, Bernard A, Clementy $\mathrm{N}$, et al. Severe recurrent vasovagal syncope and multidisciplinary rehabilitation: a prospective randomized pilot study. Int I Cardiol. 2015:187:658-9.

25. Aydin MA, Mortensen K, Salukhe TV, et al. A standardized education protocol significantly reduces traumatic injuries and syncope recurrence: an observational study in 316 patients with vasovagal syncope. Europace. 2012;14:410-5.

26. Ng J, Sheldon RS, Maxey C, et al. Quality of life improves in vasovagal syncope patients after clinical trial enrollment regardless of fainting in follow-up. Auton Neurosci. 2019;219:42-8.

27. Coakley A, Bailey A, Tao J, et al. Video education to improve clinical skills in the prevention of and response to vasovagal syncopal episodes. Int J Womens Dermatol. 2020;6:186-90.

28. Government UK. Neurological disorders: assessing fitness to drive. 2020. Available at: www.gov.uk/guidance/neurological- 
disorders-assessing-fitness-to-drive (accessed 23 February 2021).

29. Solari D, Tesi F, Unterhuber M, et al. Stop vasodepressor drugs in reflex syncope: a randomised controlled trial. Heart. 2017:103:449-55.

30. Dockx K, Avau B, De Buck E, et al. Physical manoeuvers as a preventive intervention to manage vasovagal syncope: systematic review. PLOS One. 2019;14:e0212012.

31. van Dijk N, de Bruin IG, Gisolf J, et al. Hemodynamic effects of leg crossing and skeletal muscle tensing during free standing in patients with vasovagal syncope. J Appl Physiol (1985). 2005;98:584-90

32. Schroeder C, Bush VE, Norcliffe $\amalg$, et al. Water drinking acutely improves orthostatic tolerance in healthy subjects. Circulation. 2002;106:2806-11.

33. Lu CC, Diedrich A, Tung CS, et al. Water ingestion as prophylaxis against syncope. Circulation. 2003;108:1660-5.

34. Shen WK, Sheldon RS, Benditt DG, et al. 2017 ACC/AHA/HRS guideline for the evaluation and management of patients with syncope: executive summary: a report of the American with syncope: executive summary: a report of the American on Clinical Practice Guidelines and the Heart Rhythm Society. on Clinical Practice Guidelines

Circulation. 2017;136:e25-e59.
35. Madrid AH, Ortega J, Rebollo JG, et al. Lack of efficacy of atenolol for the prevention of neurally mediated syncope in highly symptomatic population: a prospective, double-blind, randomised and placebo-controlled study. J Am Coll Cardiol. 2001;37:554-9.

36. Eldadah BA, Pechnik SL, Holmes CS, et al. Failure of propranolol to prevent til-evoked systemic vasodilatation, adrenaline release and neurocardiogenic syncope. Clin Sci (Lond). 2006;111:209-16.

37. Sheldon R, Connolly S, Rose S, et al. Prevention of Syncope Trial (POST): a randomized placebo-controlled study of metoprolol in the prevention of vasovagal syncope. Circulation. 2006:113:1164-70.

38. Sheldon RS, Morillo CA, Klingenheben T, et al. Age-dependent effect of beta-blockers in preventing vasovagal syncope. effect of beta-blockers in preventing vasovaga

39. Raj SR, Faris PD, Semeniuk L, et al. Rationale for the assessment of metoprolol in the prevention of vasovagal syncope in aging subjects trial (POST5). Am Heart J. 2016;174:89-94.

40. Sheldon R, Raj SR, Rose MS, et al. Fludrocortisone for the prevention of vasovagal syncope: a randomized placebo-controlled trial. J Am Coll Cardiol. 2016;68:1-9.

41. Lahrmann $\mathrm{H}$, Cortelli P, Hilz M, et al. EFNS guidelines on the diagnosis and management of orthostatic hypotension. Eur J Neurol. 2006;13:930-6.

42. Perez-Lugones A, Schweikert R, Pavia S, et al. Usefulness of midodrine in patients with severely symptomatic neurocardiogenic syncope: a randomised control study. J Cardiovasc Electrophysiol. 2001:12:935-8.

43. Samniah N, Sakaguchi S, Lurie KG, et al. Efficacy and safety of midodrine hydrochloride in patients with refractory vasovagal syncope Am J Cardiol 2001:88:80-3.

44. Romme It van Dik Ny Go-Schon IK et al. Effectiveness of midodrine treatment in patients with recurrent vasovagal syncope not responding to non-pharmacological treatment syncope not responding to non-pharmacolog
(STAND-trial). Europace. 2011:13:1639-47.

45. Sheldon RS, Lei L, Guzman JC, et al. A proof of principle study of atomoxetine for the prevention of vasovagal syncope: the Prevention of Syncope Trial VI. Europace. 2019;21:1733-41.

46. Lei LY, Raj SR, Sheldon RS. Pharmacological norepinephrine transporter inhibition for the prevention of vasovagal syncope in young and adult subjects: a systematic review and meta-analysis. Heart Rhythm. 2020;17:1151-8.

47. Coffin ST, Raj SR. Non-invasive management of vasovaga syncope. Auton Neurosci. 2014;184:27-32.

48. Grubb BP, Samoil D, Kosinski D, et al. Use of sertraline hydrochloride in the treatment of refractory neurocardiogenic syncope in children and adolescents. J Am Coll Cardiol. 1994:24:490-4.

49. Flevari P, Leftheriotis D, Repasos E, et al. Fluoxetine vs placebo for the treatment of recurrent vasovagal syncope with anxiety sensitivity. Europace. 2017:19:127-31.

50. ClinicalTrials.gov. The lliac Arterio-venous Fistula for Treatment of Neurally Mediated Syncope Study (SHAM-ROX NMS). ClinicalTrials.gov Identifier: NCT02388087. Available at: www.clinicaltrials.gov/ct2/show/NCT02388087 (accessed 3 June 2021)

51. Palmisano P, Zaccaria M, Luzzi G, et al. Closed-loop cardiac pacing vs. conventional dual-chamber pacing with specialized sensing and pacing algorithms for syncope prevention in patients with refractory vasovagal syncope: results of long-term follow-up. Europace. 2012;14:1038-43.

52. Johansen $\mathrm{JB}$, Bexton RS, Simonsen $E \mathrm{H}$, et al. Clinical experience of a new rate drop response algorithm in the treatment of vasovagal and carotid sinus syncope. Europace. 2000;2:245-50.

53. Palmisano P, Dell'Era G, Russo V, et al. Effects of closed-loop stimulation vs DDD pacing on haemodynamic variations an occurrence of syncope induced by head-up tilt test in older patients with refractory cardioinhibitiory vasovagal syncope: the tilt test-induced response in closed-loop stimulation multicentre, prospective, single blind, randomised study. Europace. 2018;20:859-66.

54. Brignole M, Menozzi C, Moya A, et al. Pacemaker therapy in patients with neurally mediated syncope and documented asystole: Third International Study on Syncope of Uncertain Etiology (ISSUE-3): a randomised trial. Circulation. 2012;125:2566-71

55. Brignole M, Russo V, Arabia F, et al. Cardiac pacing in severe recurrent reflex syncope and tilt-induced asystole. Eur Heart 2021:42:508-16.

56. Sutton R, de Jong JSY, Stewart JM, et al. Pacing in vasovagal syncope: physiology, pacemaker sensors and recent clinical trials. Precise patient selection and measurable benefit. Heart Rhythm. 2020:17:821-8.

57. Gopinathannair R, Salgado BC, Olshanky B. Pacing for vasovagal syncope. Arrhythm Electrophysiol Rev. 2018;7:95-102.

58. Sud S, Massel D, Klein Gl, et al. The expectation effect and cardiac pacing for refractory vasovagal syncope. Am J Med. 2007;120:54-62

59. Sutton $R$, Brignole $M$. Twenty-eight years of research permit reinterpretation of tilt-testing: hypotensive susceptibility rather than diagnosis. Eur Heart J. 2014;35:2211-2.

60. Brignole M, Auricchio A, Baron-Esquivias G, et al. 2013 ESC Guidelines on cardiac pacing and cardiac resynchronization therapy: the Task Force on cardiac pacing and resynchronisation therapy of the European Society of Cardiology (ESC). Developed in collaboration with the European Heart Rhythm Association (EHRA). Eur Heart s. 2013:34:2281-329.

61. Katritsis DG, Pokushalov E, Romanov A, et al. Autonomic denervation added to pulmonary vein isolation for paroxysmal atrial fibrillation: a randomized clinical trial. J Am coll Cardiol. 2013;62:2318-25.

62. Pokushalov $\mathrm{E}$, Romanov A, Artyomenko $\mathrm{S}$, et al. Ganglionated plexi ablation directed by high-frequency stimulation and complex fractionated atrial electrograms for paroxysmal atrial fibrillation. Pacing Clin Electrophysio. 2012;35:776-84

63. Aksu T, Guler TE, Bozyel S, Yalin K. Potential usage of cardioneuroablation in vagally mediated functional atrioventricular block. SAGE Open Med. 2019;7:2050312119836308.

64. Yao Y, Shi R, Wong T, et al. Endocardial autonomic denervation of the left atrium to treat vasovagal syncope: an early experience in humans. Circ Arrhythm Electrophysiol. 2012;5:279-86.

65. Po SS, Nakagawa H, Jackman WM. Localization of left atrial ganglionated plexi in patients with atrial fibrillation. I Cardiovasc Electrophysiol. 2009;20:1186-9.

66. Malcolme-Lawes LC, Lim PB, Wright I, et al. Characterization of the left atrial neural network and its impact on autonomic modification procedures. Circ Arrhythm Electrophysiol. 2013;6:632-40.

67. Kim MY, Sikkel MB, Hunter RJ, et al. A novel approach to mapping the atrial ganglionated plexus network by generating a distribution probability atlas. I Cardiovasc Electrophysiol. 2018;29:1624-34.

68. Pachon JC, Pachon El, Cunha Pachon MZ, et al. Catheter ablation of severe neurally meditated reflex (neurocardiogenic or vasovagal) syncope: cardioneuroablation long-term results. Europace. 2011:13:1231-42.

69. Sun $W$, Zheng $L$, Qiao $Y$, et al. Catheter ablation as a treatment for vasovagal syncope: long-term outcome of endocardial autonomic modification of the left atrium. J Am Heart Assoc 2016:5:e003471. 